\title{
Internal Model Controller to Attenuate Periodic Disturbance of a First-Order Time-Delay System*
}

\author{
Can Kutlu Yuksel, Jaroslav Busek, Silviu-Iulian Niculescu, Tomas Vyhlidal\#
}

\begin{abstract}
A controller design based on the internal model control principle is introduced as an alternative to the repetitive control structure to attenuate the periodic disturbance. Such a disturbance phenomenon is observed for example in hot rolling mills due to roll eccentricity and is considered here as a motivation example. The emphasis is on the analytic design of the filter and on the time-delay parameter within the controller. By these two components, the controller action is tuned to achieve a zero gain and synchronized time shift to compensate completely the periodic disturbance. The overall procedure is validated by simulations on a model identified from real process data.
\end{abstract}

\section{INTRODUCTION}

The paper focuses on an alternative design procedure to a repetitive control, the standard method applied to compensation of periodic disturbance of a system. The study is motivated by an application in hot rolling mills, which holds an essential part of the steel production. Their production quality directly affects the subsequent steps of the overall production and there exist many physical phenomena that compromise its end-product quality. A prominent one of them is the inherent roll eccentricity that causes periodic surface defects in the end-product. These defects can be suppressed dynamically by controlling the hydraulic associated with the roller to set its vertical position according to the surface variation. By regarding the periodic surface defects as periodic disturbance acting on the mill's output, the specific problem described here can be covered under the more general problem of systems working under periodic signals.

\section{A. Problem motivation and statement}

The overall scheme of the hot rolling process is in Fig. 1. As a rule, it consists of working rolls that are in contact with the rolled plate and the back-up rolls are actuated by the hydraulic system. Since the rolled plate thickness is measured at distance $L$ from the roll working point, the process

*This work was supported by the Czech Science Foundation project No. 21-07321S - Persistent problems of repetitive control. The work of the first and third author was also supported by a public grant overseen by the French National research Agency (ANR) as part of the Investissements d'Avenir program, through the "ADI 2020" project funded by the IDEX Paris-Saclay, ANR-11-IDEX-0003-02. The first author was also supported by the Grant Agency of the Czech Technical University in Prague, grant No. SGS20/159/OHK2/3T/12.

T. Vyhlidal (\# corresponding author), C.K. Yuksel and J. Busek are with Dept. of Instrumentation and Control Eng., Faculty of Mechanical Engineering, Czech Technical University in Prague, Technická 1902/4, 16607 Praha 6, Czech Republic, Tomas.Vyhlidalefs.cvut.cz

C.K. Yuksel and S-I Niculescu are with Université Paris-Saclay, CNRS, CentraleSupélec, Inria, Laboratoire des Signaux et Systèmes, 91192 Gif-surYvette, France, Silviu.Niculescu@l2s.centralesupelec.fr inherits a time delay $\tau=\frac{v}{L}$, where $v$ is the plate velocity, considered constant here. The transfer function between the rolling gap $H(t)$ and the measured plate thickness $h(t)$ is considered as

$$
G(s)=\frac{h(s)}{H(s)}=\frac{K}{T s+1} e^{-\tau s}
$$

where $T$ is a time constant and $K$ is a static gain representing the dynamics of the hydraulic control system. The periodic disturbance to be pre-compensated is predominantly caused by the eccentricity of the back-up roll with the angular velocity $\omega_{B}[1]$. Neglecting the higher harmonics, the disturbance force is assumed in the form

$$
d_{F}(t)=F_{a} \sin \left(\omega_{d} t+\phi\right)
$$

where $F_{a}$ is the force amplitude and $\omega_{d}=\omega_{B}$ is the excitation frequency. Note that the disturbance periodic force brought by the eccentricity of the work roll with angular velocity $\omega_{W}$ is neglected in this study. Note also that the disturbance force $d_{F}$ is projected to the $\omega_{d}$-periodic output disturbance $d$ at the measured plate thickness.

\section{B. Repetitive control}

Like hot rolling mills, many engineering applications may require a system to generate prescribed periodic signals or to keep its functionality under periodic output disturbances for reasons stemming from optimization and precision. A system's capability to reject periodic disturbances is closely related to its ability to generate periodic signals due to the fact the disturbance can be canceled by generating

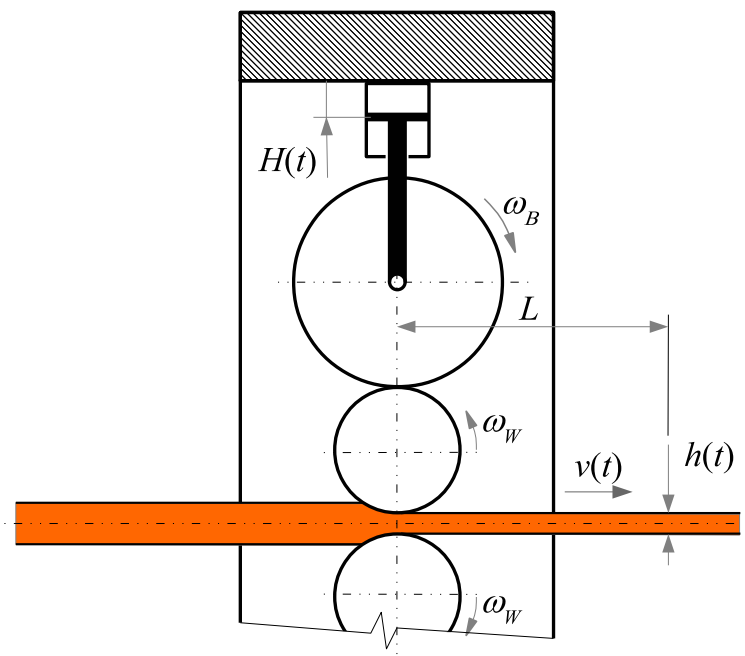

Fig. 1. Scheme of the rolling mill with work and back-up rolls. 
the opposite of it at the output. For this reason, special attention has been paid to design and improve controllers that allow various systems to generate periodic signals. Repetitive control, among all, has been the widely accepted solution to attain desired periodic behaviors.

Repetitive control is based on the Internal Model Principle (IMP), first stated by Francis and Wonham [2]. The principle states that an asymptotic tracking/rejection of a given signal can be obtained by including a model of that signal in the closed-loop control structure provided that the control system is stable. Backed by this, the implementation of the model that generates all the periodic signals (PSG) with a specific period $T_{d}$ into the controller structure laid the foundation of repetitive controllers [3].

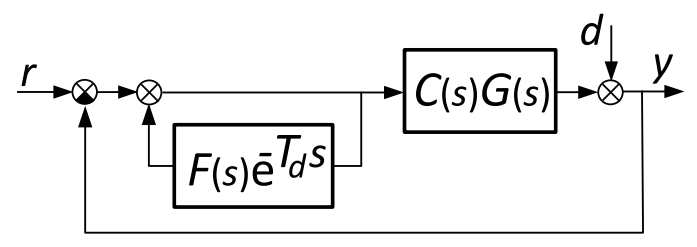

Fig. 2. Repetitive Control Scheme

The fundamental idea of repetitive control can be derived from the basic scheme in Fig. 2. The sensitivity function is given by

$$
S(s)=\frac{y(s)}{d(s)}=\frac{1-F(s) e^{-s T_{d}}}{1-F(s) e^{-s T_{d}}+C(s) G(s)} .
$$

For the ideal setting with $F(s)=1$, the transfer function $S(s)$ has infinitely many zeros $z_{k}=j \frac{2 \pi k}{T_{d}}, k \in \mathbb{Z}$, located at the stability boundary. Consequently, if the closed loop system is excited by any periodic disturbance $d$ with a period $T_{d}$ (e.g. of harmonic, square or saw nature), it is fully compensated. It results from the fact that any periodic function can be expanded into a Fourier series composed of weighted harmonics with frequencies $\omega_{k}=\frac{2 \pi k}{T_{d}} k=1,2, \ldots$ Due to the distribution of zeros, none of these harmonics passes through, as $S\left(j \frac{2 \pi k}{T_{d}}\right)=0$. However, this beneficial nature can never be achieved in practice. Assuming the open loop $C(s) G(s)$ is a strictly proper transfer function, for $F(s)=1$, the closed loop system (3) is a neutral time delay system with roots asymptotically approaching the imaginary axis at the high frequency range [4]. As this feature is a considerable stability risk, a strictly proper filter $F(s) \neq 1$ is included to the loop which turns the closed loop system to a retarded time delay system. As a consequence, however, the full compensation of the periodic signal is lost.

The first repetitive controller (RC) was designed for proper SISO systems. Following the initial design, the repetitive controller was modified with a low-pass filter to improve its applicability to strictly proper systems. Hara et al. [5] extended the modified repetitive controller to MIMO systems and gave the stability conditions for the controlled system based on small-gain theorem and theorems concerning the stability of time-delay systems. Tomizuka et al. [6] initially studied the discrete-time version of the repetitive controller. After addressing the stability problem of repetitive controllers under certain periodic input, the development of repetitive controller branched into three main topics: i) improving periodic/non-periodic tracking/rejection performance [7], ii) increasing the robustness of the controller for signals with uncertain/varying period [8], and iii) extending the application of $\mathrm{RC}$ to complicated plants such as nonlinear, MIMO, and time-delayed systems.

Despite its benefit for periodic inputs, inclusion of a PSG into the closed-loop negatively affects the control performance for non-periodic inputs. To address this issue, Inoue [9] introduced the high-order periodic signal generator in which the time-delay component $e^{-T_{d} s}$ is replaced by a polynomial $W(s)=\sum_{i=1}^{m} w_{i} e^{-m s T_{d}}$ where $w_{i}$ denotes the weight of the $i^{\text {th }}$ term. The repetitive controller that consists of a high-order PSG is referred to as the highorder repetitive controller and is the key component to most advances in modern repetitive control designs. In [7], Chang et al. proposed an alternative method to Inoue's to determine the weights of the high-order repetitive controller which results in an enhanced non-periodic response performance.

For some cases, the period of a periodic signal acting on the system can slowly vary or can be uncertain. For these particular cases, the robustness of repetitive control holds great importance for a successful control. Tsao et al. [10] and Hillerstrom et al. [11] proposed an adaptive control scheme to asymptotically track signals with unknown period. Steinbuch improved the applicability and robustness of the high-order repetitive control for such cases by introducing memory loops in [8] and later on described a unifying framework in [12] to design a high-order repetitive controller that encompasses the results from their previous works. Further on, by using a semi-definite programming method, Pipeleers et al. revealed the optimal trade-off between robustness for uncertain-period and sensitivity for non-periodic signals and unified the problem of designing a high-order repetitive control for non-periodic signals and uncertainperiodic signals [13].

The extension of repetitive control to other types of systems such as nonlinear, time-delay and MIMO systems and to systems with strict constraints gained attention as more and more applications start to be represented by such models effectively. Weiss et al. [14] presented a general repetitive control design procedure for MIMO linear plants in continuous time. For systems suffering from limited control input a solution was proposed in [15]. In [16]-[18] the stability conditions discussed in [3] were extended to nonlinear systems and in [19] Ghosh et al. described a finitedimensional SISO repetitive controller which does not posses a time-delay component for two different classes of nonlinear plants. For systems with input time-delay, Omura et al. introduced an additional time-delay in which the delay is set to be the difference between the period of the disturbance and the transport delay present in the system [1]. Their study was also motivated by the control of hot rolling mills. In [20], Guvenc et al. proposed a mapping-based repetitive control 
design method suitable for time-delay systems.

The main objective of this paper is to propose a straightforward procedure for compensation of a periodic disturbance, which is based on an internal model control scheme. At this stage, we aim at a single harmonic disturbance signal of the form (2), respectively to its projection to the measured system output. The extension to cover a more complex periodic signal will be addressed in the subsequent work.

\section{INTERNAL MODEL CONTROL BASED SCHEME}

The Internal Model Control (IMC) arrangement was first introduced and carefully studied by Garcia and Morari [21]. Its design and performance under periodic disturbances were studied in [22] and its application to first order systems with time-delay was studied by Vyhlidal and Zitek in [23], see also [24]. Due to its structure, IMC clarifies the external disturbance acting on the system and lets the controller decide its control action directly with respect to the reference and disturbance signal. This feature of IMC allows the controller to be designed explicitly with the knowledge of signals and robustness criteria. Since a transition between IMC and the classical feedback exists, the controller formed in IMC can be transformed and implemented in a classical feedback system in a straightforward manner.

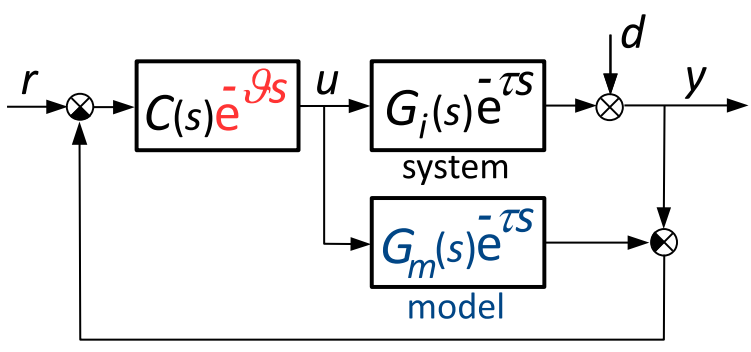

Fig. 3. Proposed internal model control scheme for periodic disturbance compensation

The modification of the standard IMC scheme to handle the periodic disturbance of a form (2) is depicted in Fig. 3. The system model, considered in the form (1), can be decomposed to the invertible part

$$
G_{i}(s)=\frac{K}{T s+1}
$$

and non-invertible part $e^{-s \tau}$. The transfer function $G_{m}(s)$ then denotes the model of the invertible part of the system dynamics. The delay $\tau$ is assumed to be known exactly and it is identical for the system and its model. The controller transfer function $C(s)$ is supplemented by an additional term with a delay $\vartheta$, which will be involved in the disturbance compensation.

The sensitivity function of the scheme in Fig. 3 is given by

$$
S(s)=\frac{y(s)}{d(s)}=\frac{1-C(s) G_{m}(s) e^{-s(\tau+\vartheta)}}{1+C(s)\left(G_{i}(s)-G_{m}(s)\right) e^{-s(\tau+\vartheta)}} .
$$

Following the design proposed in [23], the controller is given by

$$
C(s)=\frac{1}{G_{m}(s)} F(s),
$$

where $F(s)$ is a strictly proper filter satisfying

$$
\lim _{s \rightarrow 0} F(s)=1
$$

i.e. it has a unit static gain.

The IMC closed-loop structure can be transformed into a classical feedback arrangement shown in Fig. 4, with the controller

$$
\begin{aligned}
& R(s)=\frac{C(s) e^{-s \vartheta}}{1-C(s) G_{m}(s) e^{-s(\tau+\vartheta)}}= \\
& \frac{F(s) e^{-s \vartheta}}{G_{m}\left(1-F(s) e^{-s(\tau+\vartheta)}\right)} .
\end{aligned}
$$

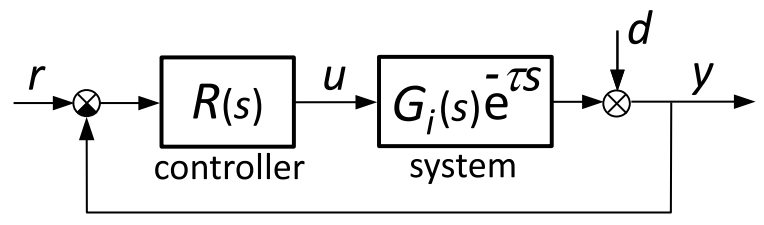

Fig. 4. Classical Feedback Arrangement

\section{A. Controller design}

The objective is to find an IMC controller such that it enables a time-delay system to asymptotically track the reference $r$ while an $\frac{2 \pi}{\omega_{d}}$-periodic signal $d$ acts on the plant's output as the external disturbance.

Assuming the model $G_{m}(s)$ and system $G_{i}(s)$ transfer functions are identical and the controller $C(s)$ is given by (6), the closed-loop transfer function given in (5) can be further simplified to

$$
S(s)=1-F(s) e^{-s(\tau+\vartheta)} .
$$

Thus, in order to cancel the periodic disturbance (2), the following condition needs to be satisfied

$$
1-F\left(j \omega_{d}\right) e^{-j \omega_{d}(\tau+\vartheta)}=0 .
$$

This equality can be split into

$$
\begin{gathered}
\left|F\left(j \omega_{d}\right)\right|=1 \\
\arg F\left(j \omega_{d}\right)-\omega_{d}(\tau+\vartheta)=2 k \pi,
\end{gathered}
$$

where $k \in \mathbb{Z}$. From (12), assuming that $\arg F\left(j \omega_{d}\right)<0$, the smallest possible (positive) delay $\vartheta$ can be determined as

$$
\vartheta=\frac{2 l \pi+\arg F\left(j \omega_{d}\right)}{\omega_{d}}-\tau
$$

where

$$
l=\left\lfloor\frac{\tau \omega_{d}-\arg F\left(j \omega_{d}\right)}{2 \pi}\right\rfloor+1 .
$$

Taking into account the above derived conditions, the overall controller can be designed in two steps. First, the 
filter satisfying the condition (11) is designed. Second step then consists of determining the delay (13).

In what follows, we propose and analyse two low-order structure filters that satisfy the above derived conditions. Taking into account the controller structure (6) and the considered first order (4) plus dead time $\tau$ dynamics, the denominator polynomial order $n$ and the numerator polynomial order $m$ of the filter $F(s)$ need to satisfy the inequality

$$
n-m \geq 1
$$

Besides, the filter needs to satisfy the condition (7).

\section{B. Second-order filter}

The simplest form of a filter that can be employed to satisfy the conditions (11), (15) and (7) is the second-order filter of the form

$$
F(s)=\frac{\Omega^{2}}{s^{2}+2 \xi \Omega s+\Omega^{2}}
$$

where $\xi$ is the damping and $\Omega$ is the natural frequency of the filter. The magnitude and the argument at the frequency response of (16) are given by

$$
\begin{gathered}
|F(j \omega)|=\frac{\Omega^{2}}{\sqrt{\left(\Omega^{2}-\omega^{2}\right)^{2}+4 \xi^{2} \Omega^{2} \omega^{2}}}, \\
\arg F(j \omega)=\operatorname{atan} \frac{-2 \Omega \xi \omega}{\Omega^{2}-\omega^{2}} .
\end{gathered}
$$

The desired condition (11) imposes

$$
\left(\Omega^{2}-\omega_{d}^{2}\right)^{2}+4 \xi^{2} \Omega^{2} \omega_{d}^{2}=\Omega^{4}
$$

which can be simplified to

$$
\Omega^{2}\left(2-4 \xi^{2}\right)=\omega_{d}^{2}
$$

yielding the positive solution

$$
\Omega=\frac{\omega_{d}}{\sqrt{2-4 \xi^{2}}},
$$

assuming $0<\xi<\frac{1}{\sqrt{2}}$. Thus the filter (16) design stems from the selection of $\xi$ from the given range and determining the natural frequency $\Omega$ from (21).

A drawback of the proposed second-order filter is its limited flexibility to tune the control system for performance and robustness. Besides, it has an oscillatory response with the damping determined by $\xi$. Thus, the filter is not optimal concerning $\mathcal{H}_{\infty}$-norm, given by

$$
\|F(j \omega)\|_{\infty}=\frac{1}{2 \xi \sqrt{1-\xi^{2}}}
$$

as $\mathcal{H}_{\infty}>1$, see Fig. 5 .

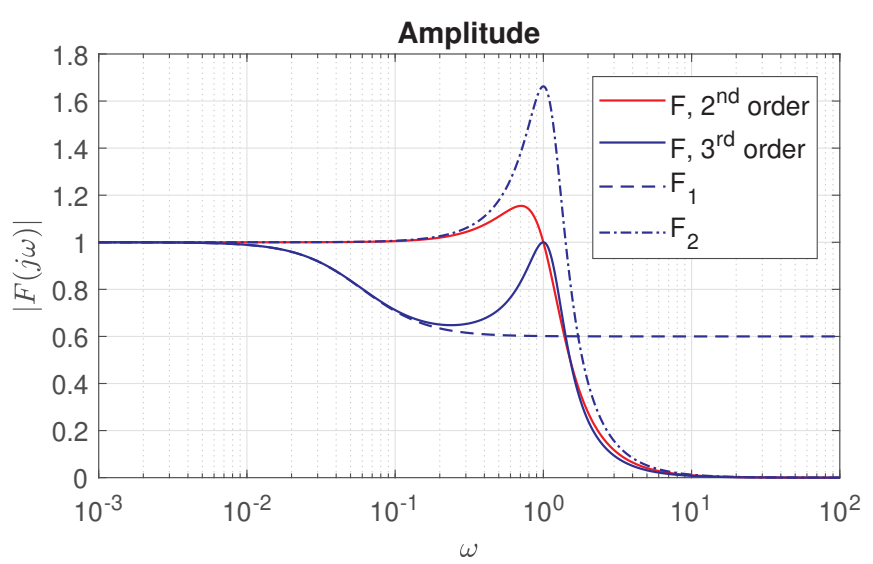

Fig. 5. Amplitude frequency response of $F(s)$ with scaled frequency of disturbance oscillations $\omega_{d}=1$ : i) second-order filter (16) with selected $\xi=0.5$ and $\Omega=1$ determined by (21); ii) third-order filter (23) composed of (24) and (25) with selected $T_{f}=6 \pi, \alpha=0.6$ and $\bar{\xi}=0.317, \bar{\Omega}=$ 1.119 determined by (32) and (29), respectively.

\section{Third-order filter}

In order to provide certain flexibility in the filer tuning, a third-order filter is proposed

$$
F(s)=F_{1}(s) F_{2}(s),
$$

composed of

$$
F_{1}(s)=\frac{\alpha T_{f} s+1}{T_{f} s+1},
$$

where the time-constant $T_{f}$ and $0<\alpha<1$ are tune-able parameters, and the second-order filter

$$
F_{2}(s)=\frac{\bar{\Omega}^{2}}{s^{2}+2 \bar{\xi} \bar{\Omega} s+\bar{\Omega}^{2}},
$$

where $\bar{\xi}$ and $\bar{\Omega}$ are the damping and natural frequency, respectively.

The magnitude of the filter (24) is given as

$$
\left|F_{1}(j \omega)\right|=\sqrt{\frac{\alpha^{2} T_{f}^{2} \omega^{2}+1}{T_{f}^{2} \omega^{2}+1}} .
$$

Since $\alpha<1$, (26) is a decreasing function with a limit

$$
\lim _{\omega \rightarrow \infty}\left|F_{1}(j \omega)\right|=\alpha .
$$

Therefore, the condition (11) needs to be met by the filter $F_{2}(s)$ given in (25). Placing the maximum magnitude of $F_{2}(j \omega)$ at $\omega_{d}$, as indicated in Fig. 5, leads to

$$
\begin{gathered}
\frac{1}{2 \bar{\xi} \sqrt{1-\bar{\xi}^{2}}}\left|F_{1}\left(j \omega_{d}\right)\right|=1, \\
\bar{\Omega}=\frac{\omega_{d}}{\sqrt{1-2 \bar{\xi}^{2}}} .
\end{gathered}
$$

The equation (28) can be expressed in the form

$$
4 \bar{\xi}^{4}-4 \bar{\xi}^{2}+\left|F_{1}\left(j \omega_{d}\right)\right|^{2}=0 .
$$

which yields the solutions

$$
\bar{\xi}_{1 . .4}= \pm \sqrt{\frac{1 \pm \sqrt{1-\left|F_{1}\left(j \omega_{d}\right)\right|^{2}}}{2}} .
$$



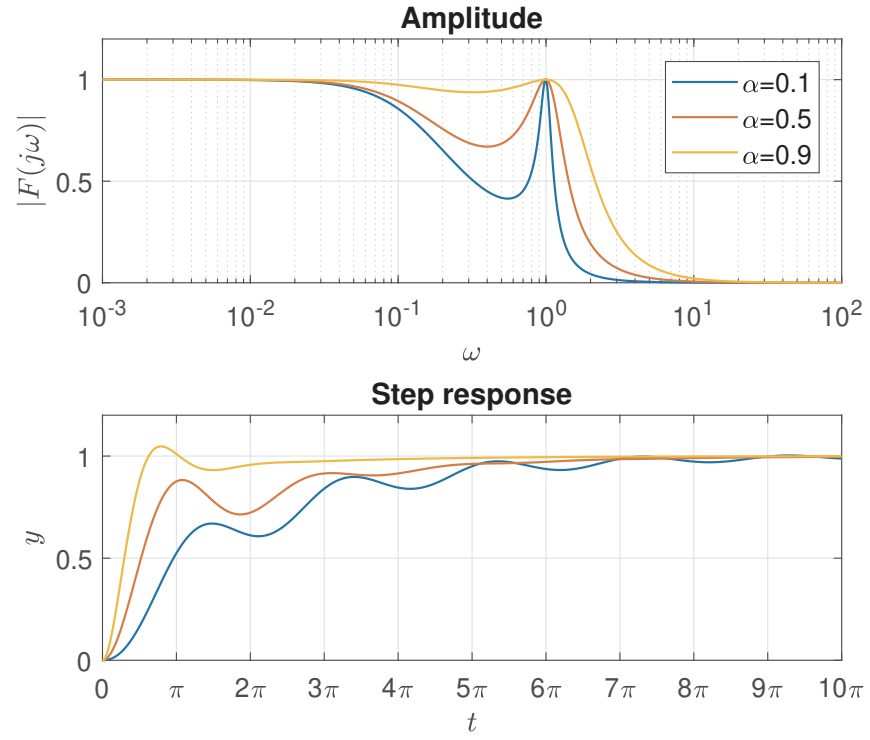

Fig. 6. Amplitude and step responses of the filter (23) for selected $\alpha$ and $T_{f}=2 \pi$

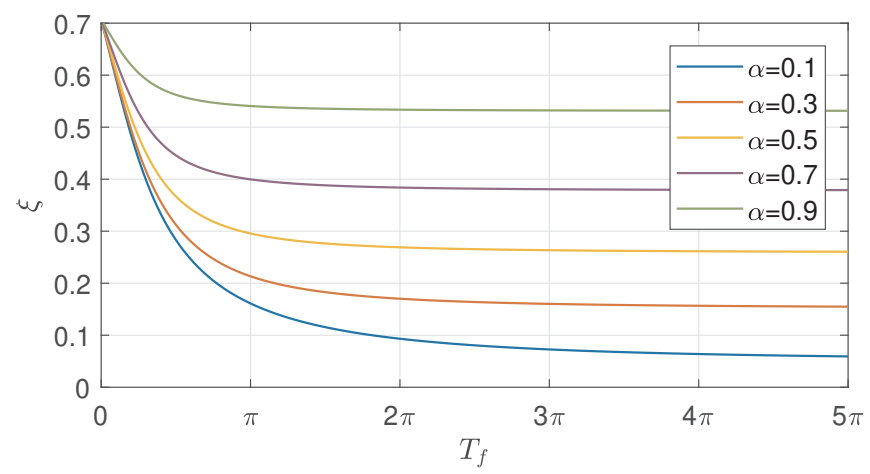

Fig. 7. Damping $\bar{\xi}$ of the filter part (25) with respect to optional parameter $T_{f}$ and $\alpha$, assuming the scaled frequency $\omega_{d}=1$

Taking into account the range $\bar{\xi} \in\left[0, \frac{1}{\sqrt{2}}\right]$ and (26), the damping of the filter part (25) is given by

$$
\bar{\xi}=\sqrt{\frac{1-T_{f} \omega_{d} \sqrt{\frac{1-\alpha^{2}}{T_{f}^{2} \omega_{d}^{2}+1}}}{2} .}
$$

The parameter $\alpha$ establishes a feature to set the optimum trade-off between the level of oscillation and the overshoot observed in the control system's response. As can be seen in Fig. 6 for scaled $\omega_{d}=1$ and selected $T_{f}=2 \pi$, higher values of $\alpha$ impose the fast control action, while smaller values impose slower response which is more affected by the oscillatory mode. Besides, the lower values of $\alpha$ result in deeper delve of $|F(j \omega)|$ to the left of $\omega_{d}$. As shown in Fig. 7 drawn again for the scaled $\omega_{d}=1$, the influence of $\xi$ on $T_{f}$ tends to diminish for $T_{f} \leq 2 \pi$. Naturally, increasing $T_{f}$ leads to more robust, but slower setting. The robustness is also enhanced by the selection of rather lower values of $\alpha$.

The argument shift needed to asses the delay (13) is given by

$$
\arg F\left(j \omega_{d}\right)=\operatorname{atan} \frac{-T_{f}(1-\alpha) \omega_{d}}{\alpha T_{f}^{2} \omega_{d}^{2}+1}+\operatorname{atan} \frac{-2 \bar{\Omega} \bar{\xi} \omega_{d}}{\bar{\Omega}^{2}-\omega_{d}^{2}}
$$

\section{VALIDATION B Y SIMULATIONS}

At this preliminary stage, a simulation based validation is performed on the transfer function (1) with parameters $T=0.8 \mathrm{~s}, K=0.5$ and $\tau=3.8 \mathrm{~s}$ identified from the data, shown in Fig. 8, measured on a rolling mill with configuration according to Fig. $1^{1}$. As can be seen from the comparison between the measured and simulated output (the rolled plate thickness $h(t)$ ), the model fits the data fairly well. The periodic system output disturbance with $\omega_{d}=5.32 \mathrm{~s}^{-1}$ $\left(T_{d}=1.18 \mathrm{~s}\right)$ and the amplitude $d_{a}=0.011 \mathrm{~mm}$ has been identified by the FFT analysis.

Two IMC controllers (6) are considered, the first with the second-order filter (16) and the second with third-order filter (23) composed of (24) and (25). For the secondorder filter, the damping $\xi=0.5$ was selected providing $\Omega=5.324 \mathrm{~s}^{-1}$ by (21). For the third-order filter, the setting $T_{f}=4 \mathrm{~s}$ and $\alpha=0.3$ were selected to gain an enhanced level of robustness at the cost of slower time responses. The two parameters of (25) evaluated as $\bar{\xi}=0.153$ and $\bar{\Omega}=5.454 \mathrm{~s}^{-1}$ are determined by (32) and (29), respectively. By (13) and (14), the controller delay values were determined as $\vartheta=0.625 \mathrm{~s}(l=4)$ for the second-order filter and $\vartheta=0.634 \mathrm{~s}(l=4)$ for the third-order filter.

The simulation results are shown in Fig. 9 for nominal model parameters $T=0.8 \mathrm{~s}, K=0.5$ and in Fig. 10 for perturbed parameters of $G_{i}(s)$ to $T_{p}=0.64 \mathrm{~s}, K_{p}=0.7$. The transient of the periodic disturbance compensation can be seen at the beginning of the response. At time $t=20 \mathrm{~s}$ the set-point $r$ changes from 0 to 0.05 . At the transients, it is clearly seen that enhanced robustness by the third-order filter was achieved.

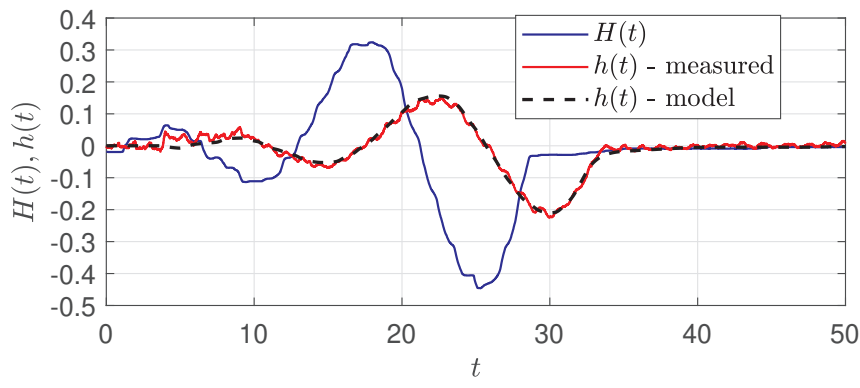

Fig. 8. Measured rolling process data and their fitting by the model $(1)^{1}$

\section{CONCLUSION}

Compared to the repetitive control strategy initiated by Inoue et al., a different approach to form a controller has been proposed and studied to fully attenuate the periodic disturbance. The introduced technique provides a fully analytic controller design that is relatively easy to implement. At

\footnotetext{
${ }^{1}$ The authors acknowledge the help of PT Solution Worldwide company for providing the data.
} 

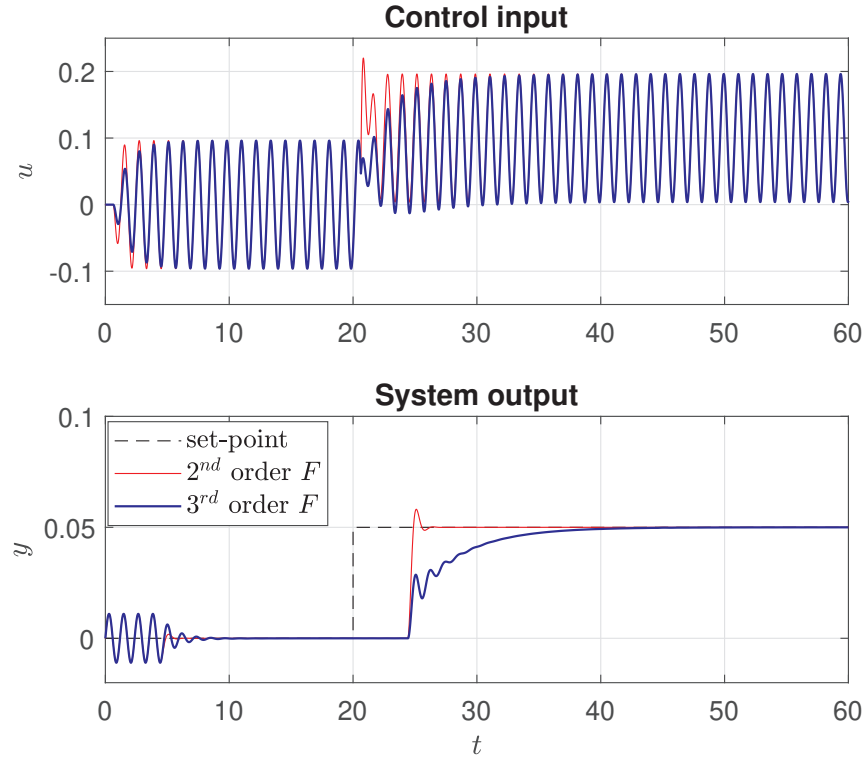

Fig. 9. Compensation of the periodic disturbance by the IMC controller (8) of system (1) with nominal parameters $\left(G_{i}(s)=G_{m}(s)\right)$
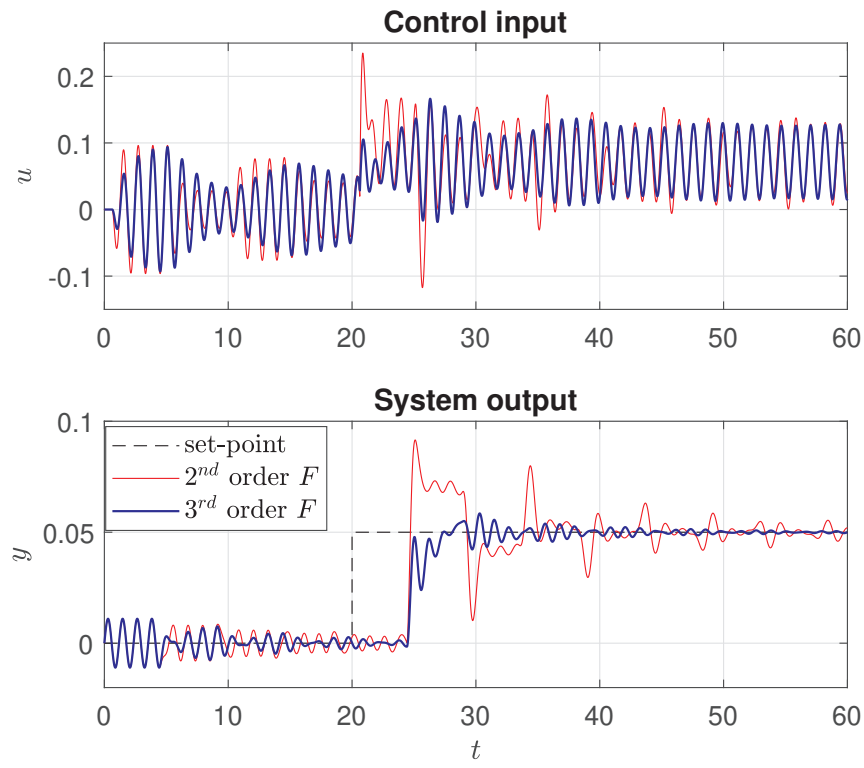

Fig. 10. Compensation of the periodic disturbance by the IMC controller (8) of system (1) with perturbed parameters $\left(G_{i}(s) \neq G_{m}(s)\right)$

this stage, the proposed method has been tested on a model identified from the data measured at the hot-rolling process. The implementation of the algorithm on an industrial controller and tests on the rolling mill application are the next steps. From a theoretical point of view, a scheme that fully compensates periodic signal that consists of several harmonic modes is to be targeted.

\section{REFERENCES}

[1] K. Omura, H. Ujikawa, O. Kaneko, Y. Okano, S. Yamamoto, H. Imanari, and T. Horikawa, "Attenuation of roll eccentric disturbance by modified repetitive controllers for steel strip process with transport time delay," IFAC-PapersOnLine, vol. 48, no. 17, pp. 131-136, 2015.

[2] B. A. Francis and W. M. Wonham, "The internal model principle of control theory," Automatica, vol. 12, no. 5, pp. 457-465, 1976.
[3] T. Inoue, M. Nakano, T. Kubo, S. Matsumoto, and H. Baba, "High accuracy control of a proton synchrotron magnet power supply," IFAC Proceedings Volumes, vol. 14, no. 2, pp. 3137-3142, 1981.

[4] J. K. Hale, S. M. V. Lunel, L. S. Verduyn, and S. M. V. Lunel, Introduction to functional differential equations. Springer Science \& Business Media, 1993, vol. 99.

[5] S. Hara, Y. Yamamoto, T. Omata, and M. Nakano, "Repetitive control system: A new type servo system for periodic exogenous signals," IEEE Transactions on automatic control, vol. 33, no. 7, pp. 659-668, 1988.

[6] M. Tomizuka, T.-C. Tsao, and K.-K. Chew, "Analysis and synthesis of discrete-time repetitive controllers," Journal of Dynamic Systems, Measurement, and Control, vol. 111, no. 3, pp. 353-358, 1989.

[7] W. S. Chang, I. H. Suh, and T. W. Kim, "Analysis and design of two types of digital repetitive control systems," Automatica, vol. 31, no. 5, pp. 741-746, 1995.

[8] M. Steinbuch, "Repetitive control for systems with uncertain periodtime," Automatica, vol. 38, no. 12, pp. 2103-2109, 2002.

[9] T. Inoue, "Practical repetitive control system design," in 29th IEEE Conference on Decision and Control. IEEE, 1990, pp. 1673-1678.

[10] T.-C. Tsao, Y.-X. Qian and, and M. Nemani, "Repetitive control for asymptotic tracking of periodic signals with an unknown period," $J$. Dyn. Sys., Meas., Control, vol. 122, no. 2, pp. 364-369, 2000.

[11] G. Hillerstrom, "Adaptive suppression of vibrations-a repetitive control approach," IEEE Transactions on Control Systems Technology, vol. 4, no. 1, pp. 72-78, 1996.

[12] M. Steinbuch, S. Weiland, and T. Singh, "Design of noise and periodtime robust high-order repetitive control, with application to optical storage," Automatica, vol. 43, no. 12, pp. 2086-2095, 2007.

[13] G. Pipeleers, B. Demeulenaere, J. De Schutter, and J. Swevers, "Robust high-order repetitive control: optimal performance trade-offs," Automatica, vol. 44, no. 10, pp. 2628-2634, 2008.

[14] G. Weiss and M. Häfele, "Repetitive control of mimo systems using $h_{\infty}$ design," Automatica, vol. 35, no. 7, pp. 1185-1199, 1999.

[15] E. Kurniawan, M. I. Afandi et al., "Repetitive control system for tracking and rejection of multiple periodic signals," in 2017 International Conference on Robotics, Automation and Sciences (ICORAS). IEEE, 2017, pp. 1-5.

[16] S. Hara, T. Omata, and M. Nakano, "Synthesis of repetitive control systems and its application," in 1985 24th IEEE Conference on Decision and Control. IEEE, 1985, pp. 1387-1392.

[17] T. Omata, S. Hara, and M. Nakano, "Nonlinear repetitive control with application to trajectory control of manipulators," Journal of Robotic systems, vol. 4, no. 5, pp. 631-652, 1987.

[18] Y. Lin, C. Chung, and T. Hung, "On robust stability of nonlinear repetitive control system: Factorization approach," in 1991 American Control Conference. IEEE, 1991, pp. 2646-2647.

[19] J. Ghosh and B. Paden, "Nonlinear repetitive control," IEEE Transactions on Automatic Control, vol. 45, no. 5, pp. 949-954, 2000.

[20] B. A. Güvenç and L. Güvenç, "Robust repetitive controller design in parameter space," Journal of Dynamic Systems, Measurement and Control, vol. 182, no. 2, pp. 406-413, 2006.

[21] C. E. Garcia and M. Morari, "Internal model control. a unifying review and some new results," Industrial \& Engineering Chemistry Process Design and Development, vol. 21, no. 2, pp. 308-323, 1982.

[22] Y.-S. Lu, "Internal model control of lightly damped systems subject to periodic exogenous signals," IEEE Transactions on Control Systems Technology, vol. 18, no. 3, pp. 699-704, 2009.

[23] T. Vyhlídal and P. Zítek, "Control system design based on a universal first order model with time delays," Acta Polytechnica, vol. 41, no. 4-5, 2001.

[24] P. Zítek, J. Bušek, and T. Vyhlídal, "Anti-windup conditioning for actuator saturation in internal model control with delays," in LowComplexity Controllers for Time-Delay Systems. Springer, 2014, pp. $31-45$. 